\title{
Stability analysis of a rotating flow toward a shrinking permeable surface in nanofluid
}

\begin{abstract}
The rotating boundary layer flow over a shrinking permeable surface in nanofluid is numerically studied. The similarity transformation is used to transform the partial differential equations into nonlinear ordinary differential equations. Later, these equations are determined by using bvp4c package in the MATLAB software. The numerical results reveal that there is more than one solution called dual solutions obtained for a certain range of the rotation and suction parameters. A stability analysis is performed to determine which solution is stable by depending on the sign of the eigenvalues. Based on this analysis, the results indicate that the upper branch solution (first solution) is linearly stable, while the lower branch solution (second solution) is linearly unstable.
\end{abstract}

Keyword : Dual solutions; Nanofluid; Rotating flow; Shrinking sheet; Stability analysis. 